\title{
La chirurgia computer-assisitita nella ricostruzione del LCA
}

\author{
S. Zaffagnini, A. Grassi, G.M. Marcheggiani Muccioli, S. Bignozzi, N. Lopomo, \\ T. Roberti Di Sarsina, F. Raggi, A. Scarale, M. Marcacci \\ Laboratorio di Biomeccanica, Istituto Ortopedico Rizzoli, Bologna
}

\begin{abstract}
The main goals of navigated procedures is to improve the correct position of the graft, and allow the possibility of performing stability testing. Our experience with CAS for ACL reconstruction includes more than 200 cases since 2004, performed with different reconstructive techniques.
\end{abstract}

\section{Introduzione}

Sono ormai passati più di 15 anni dai primi lavori sulla chirurgia computer-assistita nella ricostruzione del legamento crociato anteriore (LCA) [1], quando lo scopo principale delle procedure di navigazione era quello di ottenere il corretto posizionamento del "graft" e la sua isometria durante l'escursione articolare utilizzando riferimenti anatomici. È stato infatti dimostrato come il malposizionamento dei tunnel sia responsabile di gran parte dei fallimenti della ricostruzione del LCA [2].

Recentemente, grazie a sistemi molto più vicini alle esigenze del chirurgo e all'evoluzione dei software per la chirurgia computer-assistita per il LCA, l'interesse in questo campo è notevolmente aumentato. Attualmente, infatti, la navigazione permette l'esecuzione di test di stabilità e la misurazione delle rotazioni e traslazioni, o la scomposizione di test clinici complessi come il "pivot-shift" [3], permettendo una miglior valutazione dell' efficacia di procedure chirurgiche differenti in termini di stabilità articolare e consentendo un migliore inquadramento della lassità specifica per ogni paziente.

Grazie ai miglioramenti dei sistemi di navigazione, si è assistito nel tempo alla produzione di numerosi lavori atti a valutare l'utilizzo di tecniche ricostruttive complesse come quella a "doppio fascio". Inoltre, con l'aiuto di questa tecnologia, negli ultimi anni la conoscenza dell'anatomia e della cinematica del legamento crociato anteriore è enormemente aumentata.

\section{Descrizione della chirurgia con navigazione}

La ricostruzione del LCA assistita dal navigatore viene eseguita con approccio e strumentario tradizionali. Dopo l'esecuzione dei portali artroscopici e la conferma della lesione del LCA, vengono posizionati i "tracker" tibiale e femorale attraverso "fiches" infisse rispettivamente a livello della tibia e del femore. Non esistono indicazioni specifiche riguardo il posizionamento dei "tracker"; possono essere inseriti attraverso piccole incisioni nella pelle o per via percutanea. Bisogna fare attenzione a concedere visibilità completa degli strumenti durante i passaggi della navigazione, senza interferire però con i passaggi chirurgici. Solitamente il "tracker" tibiale viene fissato a livello dell'incisione chirurgica attraverso la quale vengono prelevati i tendini gracile e semitendinoso (qualora costituiscano il "graft" utilizzato per la ricostruzione del LCA) o a livello della tibia distale, e orientato distalmente. Il "tracker" femorale invece viene inserito al di sopra del condilo mediale, orientato distalmente rispetto al ginocchio.

Una volta posizionati i "tracker", si procede con l'acquisizione dei reperi anatomici del paziente mediante l'utilizzo di un puntatore.

A livello tibiale vengono acquisiti:

- per via percutanea:

- malleolo laterale e mediale;

- punto più mediale e laterale del piatto tibiale;

- punto di uscita dei tunnel ossei;

- per via artroscopica:
"La navigazione chirurgica permette la valutazione dell'efficacia di procedure chirurgiche differenti in termini di stabilità articolare" 


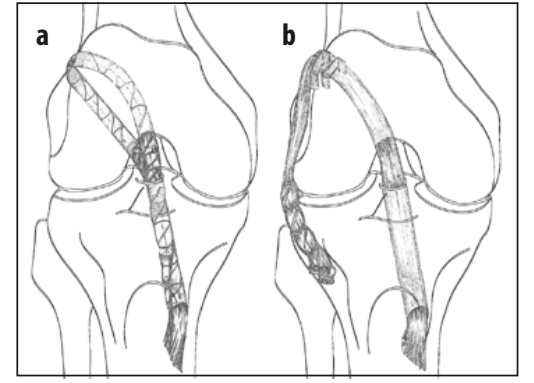

$\triangle$ Fig. 1 - Ricostruzione del LCA con gracile e semitendinoso con tecnica a doppio fascio (a) e con tecnica a singolo fascio in posizione "overthe-top" con plastica extra-articolare (b)
- punto d'ingresso dei tunnel ossei;

- inserzione del LCA;

- punto più distale del piatto tibiale laterale e mediale.

A livello femorale vengono acquisiti:

- per via percutanea:

- testa femorale (attraverso movimenti di circonduzione dell'anca);

- epicondilo mediale e laterale;

- punto di uscita dei tunnel ossei;

- per via artroscopica:

- punto più distale dei condili laterale e mediale;

- inserzione del LCA;

- punto d'ingresso dei tunnel ossei.

Una volta completata la registrazione, è possibile eseguire i test cinematici pre-operatori, durante i quali la gamba viene flessa, con la coscia sorretta da un assistente e il piede poggiato sul tavolo operatorio. Le acquisizioni cinematiche includono solitamente:

- "range-of-motion" passivo (PROM);

- valgo e varo stress (VV) a $0^{\circ}$ e $30^{\circ}$ di flessione con forza massima;

- rotazione interna ed esterna (IE) a $30^{\circ}$ e $90^{\circ}$ di flessione con forza massima;

- cassetto anteriore e posteriore (AP) a $30^{\circ} \mathrm{e}$ $90^{\circ}$ di flessione;

- "pivot-shift".

Dopo l'esecuzioni di tali manovre è possibile completare l'intervento con la ricostruzione del LCA e la nuova acquisizione di test cinematici dopo il fissaggio del "graft".

\section{La nostra esperienza clinica}

"Grazie alla navigazione è possibile inquadrare la lassità specifica per ogni paziente e di eseguire una chirurgia personalizzata"
Per valutare la lassità e la cinematica articolare abbiamo usato un sistema di navigazione ottica (BLU-IGS, Orthokey, Delaware) con un software dedicato all'acquisizione cinematica (KLEE, Orthokey, Delaware). Questo sistema applicato ci ha permesso di eseguire studi differenti, che comprendono dal 2004 la valutazione di circa 200 casi.

\section{"Double bundle" vs "single bundle"}

Gli studi clinici hanno incluso la valutazione della lassità prima e dopo la ricostruzione dell'LCA con due differenti tecniche chirurgiche utilizzate nel nostro Istituto: la prima tecnica prevede l'utilizzo dei tendini gracile e semitendinoso a doppio fascio con un singolo tunnel tibiale, passaggio in posizione "over-the-top" per il fascio antero-mediale (AM) e un tunnel femorale per il passaggio del fascio postero-laterale (PL) (Fig. 1a) [4]. La seconda tecnica prevede l'utilizzo di un "graft" a singolo fascio sempre di zampa d'oca con tunnel tibiale, passaggio in posizione "over-the-top" e una ulteriore plastica laterale fissata a livello del tubercolo del Gerdy con il capo distale del "graft" (Fig. 1b) [5].

Il confronto della lassità in AP, VV e IE tra le due tecniche non ha mostrato differenze statisticamente significative $(P>0,05)$ in tutti i test di lassità.

\section{Instabilità antero-mediale}

Risultati più interessanti sono stati ottenuti valutando pazienti operati a partire da condizioni pre-operatorie differenti. Ci siamo chiesti se fosse possibile identificare una lassità residua in pazienti con lesione combinate di LCA e legamento collaterale mediale (LCM), confrontandoli con quelli con lesione singola del LCA [6]. I pazienti sono stati prospetticamente classificati in due gruppi: i pazienti con lesione isolata del LCA sono stati usati come gruppo di controllo (Gruppo I) e i pazienti con una lesione di grado II del legamento collaterale mediale combinato con lesione dell'LCA sono stati usati come gruppo di studio (Gruppo II). I pazienti di entrambi i gruppi sono stati trattati con la ricostruzione del LCA con gracile e semitendinoso con tecnica "single-bundle" e plastica laterale. La valutazione clinica è stata effettuata usando lo "score" International Knee Documentation Committee (IKDC). La lassità pre-operatoria nei due gruppi risultava differente sia in VV sia in AP.

Il confronto post-operatorio in AP ha mostrato a $90^{\circ}$ di flessione la presenza di un certo grado di lassità nei pazienti con lesioni combinate rispetto ai pazienti con la lesione isolata del LCA. A $30^{\circ}$ di flessione, invece, le lassità post-operatorie residue non erano statisticamente differenti, confermando come il ruolo del LCM durante la traslazione antero-posteriore della tibia sia minimo in estensione, mentre diventa clinicamente più importante con il ginocchio flesso.

Un risultato analogo è stato trovato per i test in $\mathrm{VV}$, in quanto è stata rilevata una lassità residua 
nei pazienti con lesione combinata rispetto ai pazienti con lesione isolata del LCA a $30^{\circ} \mathrm{di}$ flessione, mentre in estensione non sono state trovate differenze statisticamente rilevanti tra i due gruppi, confermando come la funzione del LCM agisca maggiormente a $30^{\circ}$ di flessione rispetto alla completa estensione.

\section{Plastica laterale extra-articolare}

Il sistema di navigazione non è solo in grado di valutare lassità uniplanari, come la traslazione $\mathrm{AP}$, in VV o in IE rotazione, ma può essere usata per analisi cinematiche più dettagliate come la valutazione della traslazione AP nei compartimenti laterale e mediale o la scomposizione della rotazione e della traslazione durante la manovra del "pivot-shift". La valutazione della traslazione nei due compartimenti tibiali è risultata utile per determinare l'effetto della plastica laterale extra-articolare nel controllare la lassità articolare [7]. Infatti studi in vivo e in vitro non offrivano una interpretazione univoca circa l'effetto di una procedura extra-articolare sulla lassità del ginocchio.

Tali studi concordavano solamente nell'indicare una condivisione del carico tra le porzioni intra- ed extra-articolare della ricostruzione, evidenziando in particolare come il carico sul LCA diminuisca con l'estensione del ginocchio [8].

Con la navigazione chirurgica abbiamo valutato l'effetto di una ricostruzione extra-articolare associata alla ricostruzione del LCA con gracile e semitendinoso con tecnica a singolo fascio utilizzando la posizione "over-the-top", acquisendo la cinematica articolare di 28 pazienti.

L'acquisizione dei dati relativi alla lassità AP è stata effettuata mediante l'esecuzione dei test di Lachman e cassetto anteriore prima dell'inserimento del "graft" all'interno del tunnel tibiale. Successivamente il "graft" a singolo fascio è stato passato all'interno del tunnel tibiale e fissato sul condilo femorale laterale in posizione "over-thetop" con due cambre metalliche, dopodiché sono stati eseguiti nuovamente i test di lassità. Infine è stata effettuata una ulteriore acquisizione dopo avere eseguito una plastica extra-articolare fissando il moncone residuo del "graft" a livello del tubercolo del Gerdy con una cambra metallica, passando al di sotto della fascia lata.

Comparando la lassità del ginocchio prima e dopo la ricostruzione del LCA, è emerso come a $30^{\circ}$ di flessione il "graft" a singolo fascio abbia prodotto una riduzione della traslazione AP del compartimento mediale e laterale $(5 \mathrm{~mm})$. L'esecuzione della plastica extra-articolare invece ha prodotto una ulteriore riduzione della lassità di circa $2 \mathrm{~mm}$ esclusivamente a livello del comparto laterale ( $\mathrm{P}=0,015)$.

A $90^{\circ}$ di flessione il "graft" a singolo fascio ha prodotto una riduzione della lassità nel comparto mediale di circa $2 \mathrm{~mm}$ e nel comparto laterale di circa $5 \mathrm{~mm}$, mentre la plastica extraarticolare ha ridotto ulteriormente la traslazione $\mathrm{AP}$ in entrambi i comparti di $1 \mathrm{~mm}(\mathrm{P}<0,05)$. Una riduzione significativa è stata rilevata anche per la lassità in VV, ma non per la lassità rotazionale.

Pertanto si può affermare che, nonostante la presenza di un solo fascio, il "graft" intra-articolare sia in grado di ridurre la lassità del ginocchio, mentre la procedura aggiuntiva extraarticolare sia efficace nel controllare ulteriormente la lassità. Infatti, in estensione, il "graft" a singolo fascio riduce la traslazione AP mentre la procedura extra-articolare controlla la rotazione interna, riducendo di 1,6 $\mathrm{mm}$ la traslazione del compartimento tibiale laterale. Anche in flessione il "graft" riduce la traslazione AP della tibia, mentre la procedura extra-articolare contribuisce a controllare la traslazione tibiale riducendo la lassità di $1 \mathrm{~mm}$ in entrambi i compartimenti, producendo buoni risultati clinici [9].

\section{Doppio fascio anatomico}

La navigazione chirurgica ha permesso di valutare la cinematica articolare di 18 pazienti con lesione isolata del LCA sottoposti a ricostruzione con tecnica a doppio fascio anatomico secondo Chhabra e coll. [10]. Anche in questo caso la registrazione dei dati cinematici, attraverso i test $\mathrm{VV}$ a $0^{\circ} \mathrm{e} 30^{\circ}$, IE rotazione a $30^{\circ} \mathrm{e} 90^{\circ}$, Lachman (AP 30), cassetto anteriore (AP 90) e "pivot-shift", è stata eseguita prima e dopo la ricostruzione del LCA.

Per quanto riguarda il "pivot-shift", abbiamo analizzato la lassità articolare come la scomposizione di due parametri differenti rispetto all'angolo di flessione: traslazione AP e IE rotazione. Per ogni scomposizione abbiamo valutato l'area inclusa sotto la curva ottenuta durante i test come lassità articolare dinamica. Grazie a queste curve abbiamo anche identificato i picchi più alti e più bassi ottenuti e abbiamo registrato a quale angolo si sono verificati, descrivendo la
“L'acquisizione dei reperti anatomici e l'esecuzione dei test cinematici determina un allungamento trascurabile dei tempi chirurgici”

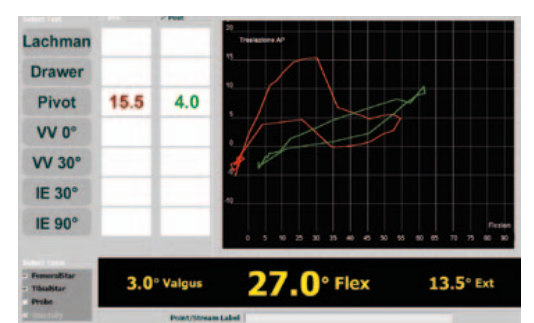

$\triangle$ Fig. 2 - Risultato del test del "pivot-shift" con il sistema BLU-IGS (Orthokey, Lewes, Delaware). La schermata mostra la traslazione antero-posteriore durante la manovra. Sulla destra è visibile il valore del picco di lassità a $30^{\circ}$, mentre nel grafico è possibile valutare la motilità passiva della tibia (pre-operatorio in rosso, post-operatorio in verde) 


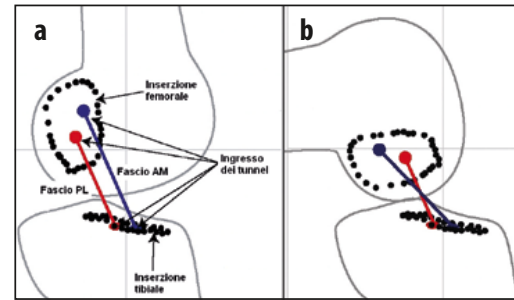

$\triangle$ Fig. 3 - Acquisizione delle inserzioni tibiale e femorale del LCA sul piano sagittale, con il ginocchio in estensione (a) $e$ in flessione (b)

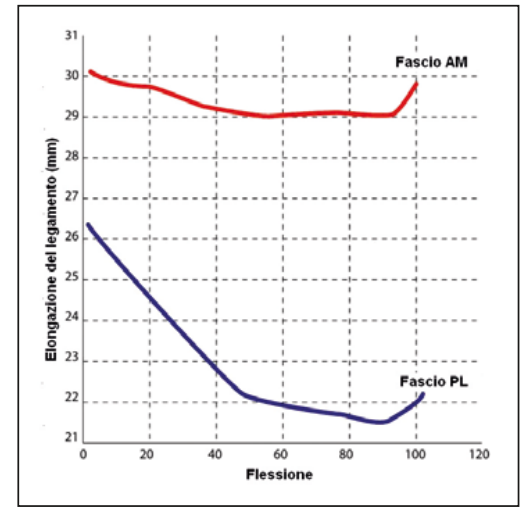

$\triangle$ Fig. 4-Allungamento dei fasci antero-mediale (AM) e postero-laterale (PL) durante la flessoestensione passiva lassità statica durante il test. Un risultato tipico di scomposizione cinematica del "pivot-shift" in traslazione AP, con software BLU-IGS, è mostrato in Figura 2.

Tutte le lassità sono risultate significativamente ridotte dopo la ricostruzione del LCA; in particolare la lassità AP ha mostrato grande riduzione durante il Lachman e il cassetto anteriore, la rotazione in $\mathrm{VV}$ ha mostrato una riduzione significativa $(\mathrm{P}<0,0001)$ sia in estensione sia a $30^{\circ}$ di flessione, mentre la riduzione dell'IE rotazione, anche se significativa $(\mathrm{P}<0,01)$, è risultata minore se paragonata agli altri test.

L'analisi dei picchi durante la manovra del "pivot-shift" ha mostrato come i più alti valori di lassità siano presenti tra $20^{\circ}$ e $30^{\circ}$ di flessione e come questi siano significativamente ridotti dopo la ricostruzione del LCA $(\mathrm{P}<0,0001)$. Questi risultati sono in accordo con quanto affermato da Bull e coll. [3], che sottolineano come il "pivot-shift" sia maggiormente discriminante nel valutare l'effetto della ricostruzione con doppio fascio nel controllo dell'instabilità dinamica dell'articolazione.

Il confronto tra le aree di lassità pre- e postoperatorie hanno sottolineato un grande recupero della stabilità dinamica dell'articolazione Da un punto di vista anatomico invece, la valutazione della posizione del "graft", mostrata in Figura 3, indica chiaramente come il neolegamento, se correttamente posizionato, abbia un comportamento simile al LCA nativo, dove i due fasci risultano paralleli in estensione e incrociati alla flessione del ginocchio. L'analisi dell'allungamento dei due fasci durante la flesso-estensione passiva ha mostrato piccole variazioni in lunghezza per il fascio AM, mentre ha evidenziato un accorciamento del PL durante la flessione (Fig. 4).

\section{Conclusioni}

Con l'aiuto di sistemi di navigazione sempre più precisi e meno invasivi, negli ultimi 15 anni la conoscenza dell'anatomia e della cinematica del LCA è aumentata enormemente. La possibilità di descrivere con grande precisione l'area di inserzione dei suoi diversi fasci e di valutare il corretto posizionamento del tunnel intra-operatoriamente può ridurre la possibilità di errore migliorando il risultato finale della ricostruzione.

Tuttavia l'uso della navigazione chirurgica per il posizionamento del tunnel rimane limitato, poiché gli obiettivi e la tolleranza per il posizionamento ottimale del "graft" sono tuttora poco compresi. Piuttosto, con l'introduzione della valutazione cinematica, diventa possibile quantificare a tempo zero l'effetto della chirurgia nel controllare la lassità del ginocchio.

Inizialmente le misure di stabilità raccolte tramite la navigazione includevano test standard uniplanari. Con il passare del tempo sono stati studiati movimenti patologici più complessi come quelli riscontrati durante la manovra del "pivot-shift".

Questi dati iniziano a definire parametri chirurgici per le varie tecniche di ricostruzione del LCA. Pertanto con queste informazioni disponibili intra-operatoriamente è ora possibile pensare a una chirurgia personalizzata per ogni singolo paziente, dove le informazioni quantitative possono aiutare ad affinare il risultato chirurgico.

\section{Bibliografia}

1. Wetzler MJ, Bartolozzi AR, Gillespie MJ et al (1996) Revision anterior cruciate ligament reconstruction. Oper Tech Orthop 6:181-189

2. Dessenne V, Lavallée S, Julliard R et al (1995) Computer-assisted knee anterior cruciate ligament reconstruction: first clinical tests. J Image Guid Surg 1:59-64

3. Bull AM, Andersen HN, Basso O et al (1999) Incidence and mechanism of the pivot shift. An in vitro study. Clin Orthop 363:219-231

4. Marcacci M, Molgora AP, Zaffagnini S et al (2003) Anatomic double-bundle anterior cruciate ligament reconstruction with hamstrings. Arthroscopy 19:540-546

5. Marcacci M, Zaffagnini S, Iacono F et al (1998) Arthroscopic intra- and extra-articular anterior cruciate ligament reconstruction with gracilis and semitendinosus tendons. Knee Surg Sports Traumatol Arthrosc 6:68-75

6. Zaffagnini S, Bignozzi S, Martelli S et al (2007) Does ACL reconstruction restore knee stability in combined lesions?: An in vivo study. Clin Orthop 454:95-99
7. Bignozzi S, Zaffagnini S, Lopomo N et al (2009) Does a lateral plasty control coupled translation during antero-posterior stress in single-bundle ACL reconstruction? An in vivo study. Knee Surg Sports Traumatol Arthrosc 17:65-70

8. Carson WG Jr (1988) The role of lateral extra-articular procedures for anterolateral rotatory instability. Clin Sports Med 7:751-772

9. Marcacci M, Zaffagnini S, Iacono F et al (2003) Intra- and extra-articular anterior cruciate ligament reconstruction utilizing autogeneous semitendinosus and gracilis tendons: 5-year clinical results. Knee Surg Sports Traumatol Arthrosc 11:2-8

10. Chhabra A, Starman JS, Ferretti M et al (2006) Anatomic, radiographic, biomechanical, and kinematic evaluation of the anterior cruciate ligament and its two functional bundles. J Bone Joint Surg Am 88[Suppl. 4]:2-10 